Objective: We describe a series of nested life course models that correspond to the critical period, accumulation, and social mobility models and test them simultaneously, on multiple CVD risk factors in, a large cohort study.

Design: Prospective birth cohort study.

Setting: England, Scotland, and Wales

Participants: 5362 singleton births in the MRC National Survey of Health and Development, followed up since their birth in 1946.

Main Outcomes: CVD risk factors at 53 years: body mass index (BMI), systolic and diastolic blood pressure, total cholesterol, lowdensity lipoprotein, high-density lipoprotein, triglycerides, glycated haemoglobin (HBA1c)

Results: Social class, according to the Registrar General's classifications, at 3 time points were utilised: childhood (father's occupation when cohort member was age 4), early adulthood (own occupation at age 26 years), and later adulthood (own occupation at 43 years). Partial F-tests comparing a saturated model with each simpler life course model were used to identify the most appropriate model for each risk factor. For women, SEP generally affected the CVD risk factors in a cumulative manner; while SEP in childhood was the prominent model for men. For example, in women BMI increased by $1.11 \mathrm{~kg} / \mathrm{m}^{2}$ (95\% CI 0.76 to 1.46$)$ per unit increase in SEP accumulation score. In men BMI was $0.42 \mathrm{~kg} / \mathrm{m}^{2}$ (0.17 to 0.68$)$ higher in those from a manual social class in childhood. In both genders, a late adulthood critical period for HBA1c was the best fitting model. BMI at age 53 reduced the associations for all outcomes but whereas BMI at age 53 captured women's lifetime BMI trajectory, it was men's BMI at earlier ages that explained more of the association than BMI at older ages. Exercise, total energy and fat intake, and menopausal status (women only) attenuated the SEP/BMI association in both genders, while lifetime smoking pattern increased the association in women (regression coefficients final model: women $0.77 \mathrm{~kg} / \mathrm{m}^{2}$ (0.39 to 1.15) and men $0.75 \mathrm{~kg} / \mathrm{m}^{2}$ (0.16 to 1.35$)$.

Conclusion: SEP across life influences CVD risk factors differently in men and women. Health behaviours may influence BMI and subsequently the other CVD risk factors, but at different points in the life course depending on gender. Gender difference in health behaviours, reproductive characteristics, and social roles across life may explain the differential effects of SEP on CVD risk factors.

\section{SOCIAL INFLUENCES ON TRAJECTORIES OF SELF-RATED HEALTH: A COMPARATIVE STUDY OF FOUR OECD COUNTRIES}

${ }^{1} \mathrm{~A}$ Sacker, ${ }^{2} \mathrm{D}$ Worts, ${ }^{2} \mathrm{P}$ McDonough. ${ }^{1}$ Institute for Social and Economic Research, University of Essex, Colchester, Essex, UK; ${ }^{2}$ Dalla Lana School of Public Health, University of Toronto, Toronto, ON, Canada

doi:10.1136/jech.2009.096727w

Objective: To describe average national trajectories of self-rated health over a 7-year period, identify social determinants of crosssectional and longitudinal health; and compare cross-national patterns.

Design: Prospective nationally representative household panel studies (the US Panel Study of Income Dynamics; British Household Panel Survey; the German Socio-Economic Panel Survey; the Danish panel from the European Community Household Panel Survey).

Setting: The US, Britain, Germany and Denmark.

Participants: Household heads and their partners of working age throughout follow-up (US: 4855; Britain: 4365; Germany: 4694; Denmark: 3252).

Main Outcome Measure: Repeated measures of self-rated health (1995-2001). Social indicators include education, occupational class, employment status, income, age, gender, minority status and marital status, all measured in 1994.
Methods: Latent growth curve models describe average national trajectories of self-rated health and individual differences in these trajectories. Latent factors representing intercept and slope components are extracted from seven annual observations across time for self-rated health, and are conditioned on predictors measured one year prior to baseline. Aging-vector graphs are used to visualise trajectories of self-rated health.

Results: The vector graphs for the US and Germany show that selfrated health remained relatively stable for young adults, declined as adults became middle aged and then became more stable again. The graphs for Britain and Denmark indicate a steady decline throughout working life. The Danish model indicates an unfavourable trend in self-rated health during a period that experienced a move to monetarism: ratings were lower for persons of a given age in 2001 than for persons of the same age in 1995. Social covariates predicted baseline health in all four countries, with the strength of association consistent with theories of welfare regime typologies. The strongest social gradients were seen in the US, while the weakest were seen in Denmark and Germany. Britain occupied a position between these two extremes. Once inequalities in baseline health had been accounted for, there were few determinants of mean health decline. There was little difference in the aging trajectories for those with advantaged and average social profiles. By contrast, disadvantage had a strong effect on aging trajectories. Differences were already apparent at 25 years of age in the US and Britain and gaps widened with age in all four countries.

Conclusion: National differences in self-rated health trajectories and their social correlates may be attributed, in part, to welfare policies.

\section{Health service research and evidence based practice}

\section{MIND THE GAP: USE OF EVIDENCE IN COMMENTARIES ON MIMR IN PROFESSIONAL JOURNALS (1988-2007)}

${ }^{1} \mathrm{~S}$ Hilton, ${ }^{1} \mathrm{~K}$ Hunt, ${ }^{1} \mathrm{M}$ Langan, ${ }^{2} \mathrm{~V}$ Hamilton, ${ }^{3} \mathrm{M}$ Petticrew, ${ }^{4} \mathrm{H}$ Bedford. ${ }^{1} \mathrm{MRC}$ Social and Public Health Sciences Unit, Glasgow, UK; ${ }^{2}$ University of Glasgow, Glasgow, UK; ${ }^{3}$ London School of Hygiene and Tropical Medicine, London, UK: ${ }^{4}$ UCL Institute of Child Health, London, UK

doi:10.1136/jech.2009.096727x

Objective: To examine how journals and magazines disseminate research evidence and guidance on best practice to health professionals by analysing the alignment between commentaries on MMR evidence in journals, and key events in the MMR controversy.

Design: Content analysis of published commentaries on MMR (1988-2007).

Data Sources: Commentaries and articles in six commonly-read UK publications aimed at community health practitioners, identified through interviews with health visitors, practice nurses, GPs and medical librarians $(n=20)$, and through a survey conducted at the Community Practitioners and Health Visiting Association Annual Conference in 2007

Main Outcome Measures: Number of comment pieces by publication, year and article type; trends in the focus, and tone (positive, neutral, negative, mixed) and whether recommendations on MMR were included.

Results: 860 articles met the inclusion criteria (British Medical Journal $n=104$, Community Practitioner $n=45$, Health Visitor $\mathrm{n}=24$, Practice Nurse $\mathrm{n}=61$, Nursing Standard $\mathrm{n}=61$ and Pulse $\mathrm{n}=565)$. Of these, 264 (31\%) made some reference to evidence endorsing the safety of MMR. Around one in ten were rated as negative $(10.9 \%, n=29)$ or neutral $(11.3 \%, n=30)$ in relation to 